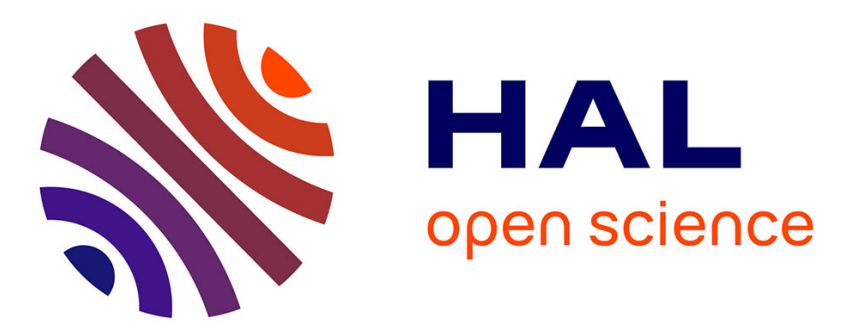

\title{
A two-step EMG-and-optimization process to estimate muscle force during dynamic movement
}

David Amarantini, Guillaume Rao, Eric Berton

\section{To cite this version:}

David Amarantini, Guillaume Rao, Eric Berton. A two-step EMG-and-optimization process to estimate muscle force during dynamic movement. Journal of Biomechanics, 2010, 43 (9), pp.1827-1830. 10.1016/j.jbiomech.2010.02.025 . hal-01442061

\section{HAL Id: hal-01442061 \\ https://hal.science/hal-01442061}

Submitted on 15 Nov 2017

HAL is a multi-disciplinary open access archive for the deposit and dissemination of scientific research documents, whether they are published or not. The documents may come from teaching and research institutions in France or abroad, or from public or private research centers.
L'archive ouverte pluridisciplinaire HAL, est destinée au dépôt et à la diffusion de documents scientifiques de niveau recherche, publiés ou non, émanant des établissements d'enseignement et de recherche français ou étrangers, des laboratoires publics ou privés. 


\title{
A two-step EMG-and-optimization process to estimate muscle force during dynamic movement
}

\author{
David Amarantini ${ }^{\mathrm{a}, *}$, Guillaume Rao ${ }^{\mathrm{b}}$, Eric Berton ${ }^{\mathrm{b}}$ \\ a Université de Toulouse, UPS, LAPMA, 118 Route de Narbonne, 31062 Toulouse Cedex 9, France \\ ${ }^{\mathrm{b}}$ Université de la Méditerranée, CNRS, Institut des Sciences du Mouvement E.J. Marey, 163 Avenue de Luminy, 13288 Marseille Cedex 09, France
}

\section{A R T I C L E I N F O}

\section{Article history:}

Accepted 15 February 2010

\section{Keywords:}

Muscle force

Muscle group moments

Cocontraction

Electromyography

Min/max optimization

Dynamic condition

\begin{abstract}
A B S T R A C T
The present study proposed a two-step EMG-and-optimization method for muscle force estimation in dynamic condition. Considering the strengths and the limitations of existing methods, the proposed approach exploited the advantages of min/max optimization with constraints on the contributions of the flexor and extensor muscle groups to the net joint moment estimated through an EMG-to-moment approach. Our methodology was tested at the knee joint during dynamic half squats, and was compared with traditional min/max optimization. In general, results showed significant differences in muscle force estimates from EMG-and-optimization method when compared with those from traditional min/ max optimization. Muscle forces were higher - especially in the antagonist muscles - and more consistent with EMG patterns because of the ability of the proposed approach to properly account for agonist/antagonist cocontraction. In addition, muscle forces agree with mechanical constraints regarding the net, the agonist, and the antagonist moments, thus greatly improving the confidence in muscle force estimates. The proposed two-step EMG-and-optimization method for muscle force estimation is easy to implement with relatively low computational requirements and, thus, could offer interesting advantages for various applications in many fields, including rehabilitation, clinical, and sports biomechanics.
\end{abstract}

(c) 2010 Elsevier Ltd. All rights reserved.

\section{Introduction}

The knowledge of the force developed by muscles during dynamic activity could be of primary interest especially in biomechanics. Due to the very slight possibility of in vivo measurements, muscle force estimation remains a major challenge despite numerous existing methods (see Erdemir et al., 2007). Static optimization methods can lead to biased muscle force, especially because those of antagonist muscles are erroneously set to zero (Challis, 1997). Forward dynamics methods (Spagele et al. 1999; Neptune et al., 2000; Seth and Pandy, 2007) increase confidence in muscle force estimates but at high complexity and computational costs, thereby reducing the potential for clinical applications (Erdemir et al., 2007). Pure optimization techniques with appropriate search strategies and algorithms have demonstrated their potential to improve the accuracy of muscle force estimates (e.g., Rasmussen et al., 2001). However, studies have highlighted the need to incorporate electromyography (EMG) in the optimization problem formulation to provide reliable redundant information on muscular activity (e.g., Amarantini and Martin, 2004; Dowling, 1997; Vigouroux et al., 2007). Additional information about

\footnotetext{
* Corresponding author. Tel.: +33 5615583 82; fax: +33 561558280 .

E-mail address: david.amarantini@cict.fr (D. Amarantini).
}

force-length and force-velocity relationships (Olney and Winter, 1985; Buchanan et al., 2004) is also required to gain more confidence in muscle force estimates in dynamic condition.

This study aimed at proposing a two-step EMG-and-optimization method to provide muscle force in dynamic condition while properly accounting for agonist-antagonist cocontraction. The first step was to estimate the contributions of the flexor and extensor muscle groups to the net joint moment using a clinically applicable EMG-to-moment approach (Centomo et al., 2007; Rao et al., 2009). The novelty of the proposed method lies in the second step, which exploited the advantages of $\mathrm{min} / \mathrm{max}$ optimization with equality constraints imposed on agonist and antagonist muscle moments to estimate muscle force. The results obtained at the knee joint using this twostep EMG-and-optimization approach were compared with those from traditional min/max optimization during half squats. We hypothesized higher force in the antagonist muscles with the proposed method because the presence of cocontraction would be properly taken into account.

\section{Methods}

The proposed approach was tested using data from a single male participant (age: 28years, height: $1.77 \mathrm{~m}$, mass: $73 \mathrm{~kg}$ ) performing seven consecutive half squats at self-selected speed, loaded with $20 \%$ body weight. This closed chain 
exercise was chosen as the experimental task because of possible applications of this study especially in rehabilitation (Rao et al., 2009).

The first step of the proposed methodology aimed at computing the net joint moment as well as contributions of the agonist and antagonist muscle groups to the net joint moment. The required input data were similar to those summarized in Amarantini and Martin (2004) (Fig. 1), but the isometric calibration was directly incorporated into the routine used for dynamic conditions for simplified and enhanced clinical applicability (Centomo et al., 2007; Rao et al., 2009). Briefly, inputs to the model were force plate data (AMTI, $240 \mathrm{~Hz}$ ), sagittal kinematics of the ankle, knee, and hip joints (Vicon, $120 \mathrm{~Hz}$ ), and EMG from the gastrocnemius medialis (GA), biceps femoris (BF), rectus femoris (RF), and vastus medialis (VM) muscles (BIOPAC MP150, $1000 \mathrm{~Hz}$ ). Interestingly at this step, the proposed method allows for estimating agonist and antagonist muscle group moments using other models (e.g., Billot et al., 2009; Doorenbosch and Harlaar, 2003).

In the second step, muscle force estimation was formulated as a new constrained $\mathrm{min} / \mathrm{max}$ optimization problem designed to produce a solution that "distributes the collaborative muscle forces in such a way that the maximum relative muscle force is as small as possible" (Rasmussen et al., 2001) while accounting for agonist-antagonist cocontraction. The input parameters included moment-arms and physiological cross-sectional area (PCSA) while the equality constraints were imposed on the agonist and antagonist muscle moments obtained from first step: find $t_{i}$

that minimizes $C\left(t_{i}\right)=\max \left(\frac{t_{i}}{\operatorname{PCSA}_{i}}\right), \quad i \in N^{*}, \quad i=1, \ldots, p$

subject at each time instant to $\left\{\begin{array}{c}t_{i}>0 \\ t_{i}<\sigma_{\max } \mathrm{PCSA}_{i} \\ x=m \\ \sum_{x=1}^{x=m}\left(r_{x} t_{x}\right)=M_{\mathrm{Kflex}} \\ \sum_{y=1}^{y=n}\left(r_{y} t_{y}\right)=M_{\text {Kext }}\end{array}\right.$

where $t_{i}$ and $\mathrm{PCSA}_{i}$ are, respectively, the force and the cross-sectional area (Visser et al., 1990) of muscle $i, \sigma_{\max }$ is the maximum muscle stress, set constant for all muscles to $40 \mathrm{~N} / \mathrm{cm}^{2}$ (Prilutsky and Gregor, 1997); $p, n$, and $m$ are, respectively, the total number of muscles considered (9), the number of knee flexors (biceps femoris short and long heads, semi-membranous, semi-tendinous, gastrocnemius), and knee extensors (vastus lateralis, medialis, and intermedius and rectus femoris). $M_{\text {Kflex }}$ and $M_{\text {Kext }}$ are the contributions of the flexor and extensor muscle group, respectively, to the knee net joint moment estimated in the first step of the proposed method and $r_{i}$ is the moment-arm of muscle $i$ (Visser et al., 1990).

The results by this EMG-and-optimization approach were compared to those obtained by using a traditional min/max optimization method (1) with the constraints on the knee net joint moment $\left(M_{K}\right)$ only:

$\left\{\begin{array}{l}0<t_{i}<\sigma_{\max } \mathrm{PCSA}_{i} \\ \sum_{i=1}^{i=p}\left(r_{i} t_{i}\right)=M_{K}\end{array}, \quad i \in N^{*}, \quad i=1, \ldots, p\right.$

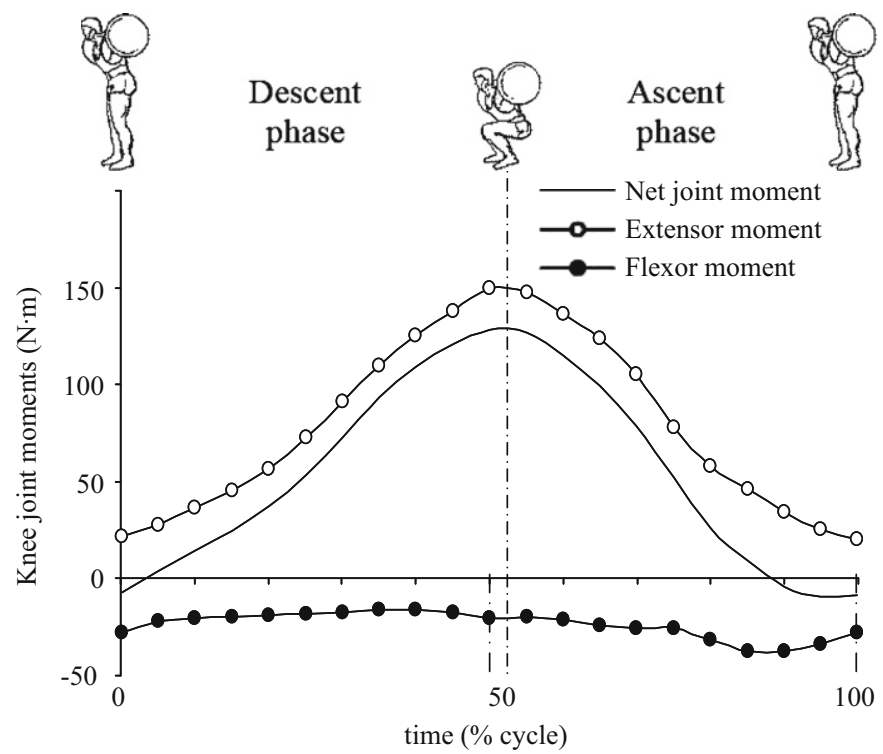

Fig. 1. From top to bottom: schematic representation of movement phases (descent/ascent); patterns of the net, flexor, and extensor moments acting at the knee joint during dynamic half squat.

\section{Results}

The squat cycle phases and the net, flexor, and extensor moments acting at the knee joint are presented in Fig. 1.

Both the traditional and the EMG-and-optimization approaches provided force estimates below the maximum force of the investigated muscles acting across the knee.

Using the traditional min/max optimization (Figs. 2a-b), force estimates for the muscles agonist to the net joint moment (knee extensors; Fig. 2a) were consistent with observed EMG from RF and VM (not presented here; see Isear et al. (1997) for typical EMG traces during squat), whereas the forces of the antagonist muscles (knee flexors) were predicted to be null during almost the entire squat cycle (Fig. 2b).

The patterns of the agonist muscle (knee extensors) forces were qualitatively similar between EMG-and-optimization and traditional $\mathrm{min} / \mathrm{max}$ optimization. However, the normalized increase of force was $13.69 \pm 11.19 \%$ (overall mean value; Fig. 2c vs. Fig. 2a), with most notable increase for the VL $(28.45 \pm 12.44 \%)$ compared with the RF, VM, and VI ( $8.75 \pm 4.38 \%, 8.84 \pm 4.41 \%$, and $8.71 \pm 4.35 \%$, respectively). Regarding antagonist muscles (knee flexors), EMG-and-optimization solutions were considerably different from those by traditional min/max optimization (Fig. 2d vs. Fig. 2b), with muscle forces by the EMG-and-optimization approach being very consistent with observed EMG from GA and BF muscles. Whatever the phase of movement, no antagonist muscle force was incorrectly set to zero. As expected from Eq. (2), the sum of individual muscle moments equaled simultaneously the knee net joint moment and the muscle group moments, thus being capable of accounting for agonist/antagonist cocontraction in muscle force estimation.

\section{Discussion}

This study was aimed at developing a two-step EMG-andoptimization process to estimate muscle force in dynamic condition. The novelty and originality of the method was the introduction of equality constraints on agonist and antagonist muscle moments estimated through an EMG-to-moment optimization procedure (Centomo et al., 2007; Rao et al., 2009). A comparison of our results was made with those from traditional $\mathrm{min} / \mathrm{max}$ optimization to evaluate the ability of the proposed method to estimate muscle force and to fulfill the specific purpose of taking properly into account agonist/antagonist cocontraction.

The two different approaches provided force estimates for all the 9 knee muscles considered in the present study, even for those from which EMG was not recorded. Qualitatively similar patterns of agonist muscle forces were found, with higher magnitudes obtained from the EMG-and-optimization approach. Such a difference could be explained by significant improvements in the estimation of antagonist muscle forces, and thus by the ability of the EMG-and-optimization approach to adequately account for agonist/antagonist cocontraction. As expected from Eq. (3), the sum of individual muscle moments from traditional min/max optimization equaled the knee net joint moment but differed from the muscle group moments, with forces inadequately set to zero without consistency with EMG of the knee flexors. On the contrary, antagonist muscle forces from EMG-and-optimization optimization were most consistent with EMG patterns, without EMG being directly enforced as a constraint in the $\mathrm{min} / \mathrm{max}$ optimization problem. EMG was taken only as an indicator of muscle activity to provide input data in the first step dedicated to the estimation of muscle group moments (see Eqs. (1) and (2); Amarantini and Martin, 2004; Centomo et al., 2007; Rao et al., 2009). Even if the EMG-and-optimization approach incorporates 
a

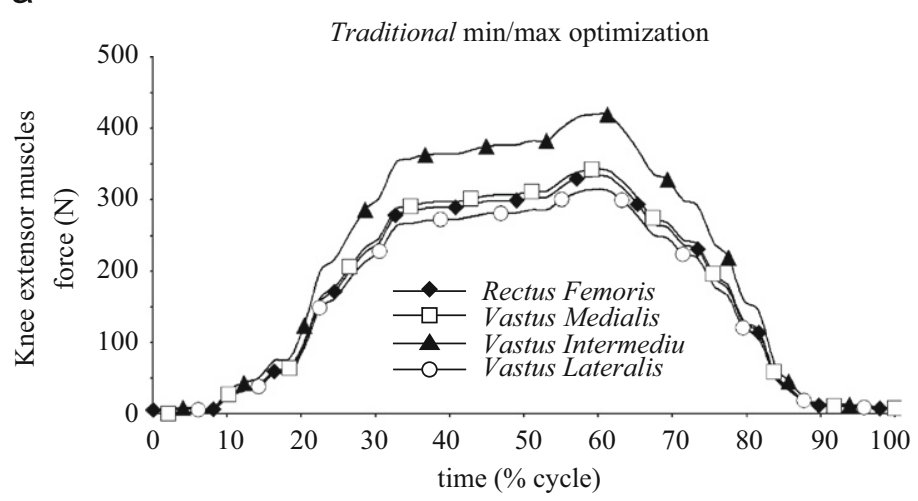

b

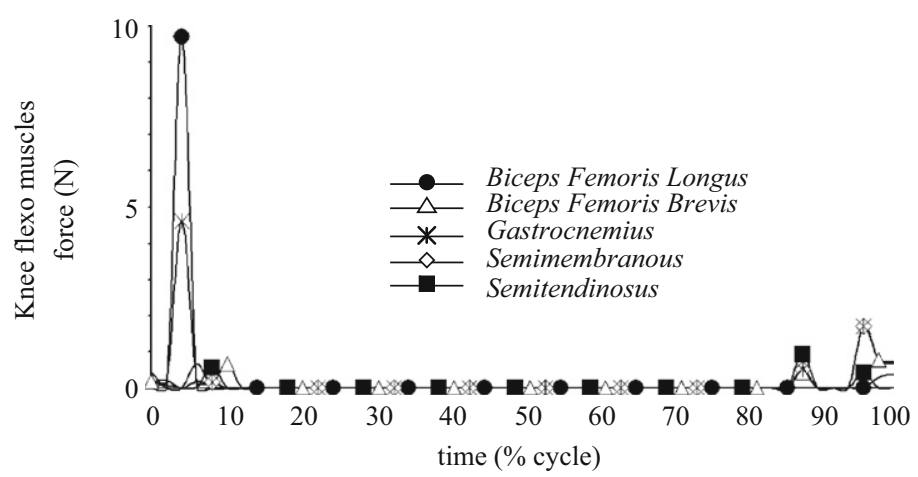

C

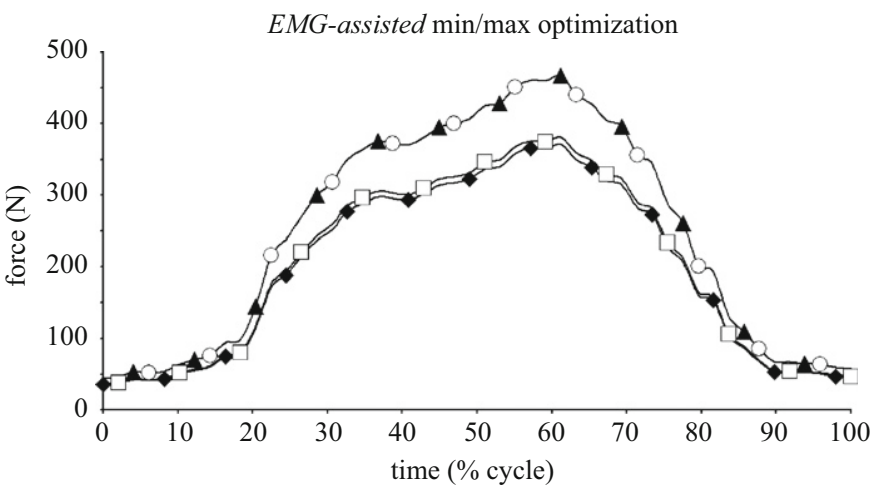

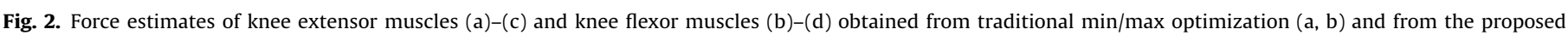
EMG-assisted min/max optimization (c, d) during dynamic half squat.

the strength of $\mathrm{min} / \mathrm{max}$ optimization in force-sharing (Rasmussen et al., 2001) and provides muscle forces compatible with VL muscle dominance (Pincivero et al., 2006; 2008), our results do not definitively resolve the question of appropriateness of force distribution within muscles. In the first step, the proposed method may benefit from more precise modeling of the forcegenerating potential of muscles. In the second step, it could incorporate PCSA values depending on joint angles (Narici et al., 1996) or pennation angles (Manal et al., 2006), different for each muscle (Buchanan, 1995), to enhance the accuracy of forcesharing solutions. However, an interesting feature of the proposed two-step approach is to reduce the sensitivity of muscle group moments to muscle parameters (Raikova and Prilutsky, 2001), thus providing more reliable equality constraints on $\min / \max$ optimization in the second step.

The results emphasize that pure traditional min/max optimization can produce misleading muscle force estimates because cocontraction remains hidden in the net joint moment. On the contrary, pure $\mathrm{min} / \mathrm{max}$ optimization with equality constraints on the agonist and antagonist moments computed in a first step using EMG-to-moment optimization (Centomo et al., 2007; Rao et al., 2009) greatly improves our confidence in muscle force estimation. The solutions by the two-step EMG-and-optimization approach indicate remarkable correspondence with both observed and reported (Isear et al., 1997) EMG patterns. The formulation of the proposed method adequately exploited min/ max optimization to properly account for cocontraction.

The proposed method extends the EMG-to-moment model originally developed by Amarantini and Martin (2004) and enables estimation of muscle force during dynamic movement treated as 2D motion. Such reduction of movement dimensionality is reasonable during half squats (Toutoungi et al., 2000) but our force-sharing solutions might not match those of the "real"
3D musculoskeletal system because the key point is the mapping between the solution-space of the optimization problem and the number of degrees of freedom of the musculoskeletal system (Jinha et al., 2006). Nevertheless, the proposed approach is not restricted to $2 \mathrm{D}$ analyses. Extension to $3 \mathrm{D}$ can be performed by incorporating additional EMG (e.g., Lloyd and Besier, 2003) and moment equilibrium constraints (Cholewicki and McGill, 1994, 1995) to provide mechanically valid optimal solutions for 3D models.

Other improvements can be expected to enhance the accuracy of muscle force estimates using the proposed method. Another criterion than minimization of muscle stress (Eq. (1)) can be used to best represent the neural command and dynamic optimization (Chao and Rim, 1973) can be incorporated - to the detriment of computational cost - to obtain muscle force that best reproduces the measured motion. Even if static and dynamic optimization may produce comparable results (Anderson and Pandy, 2001), a direction for future research could be a comparison of muscle forces from EMG-and-optimization with those from forward dynamics to address questions of validity and sensitivity of force-sharing solutions. An interesting complement would also be to compare the proposed method with other EMG-assisted processes (e.g., Cholewicki and McGill, 1994) to help determine the best way to implement EMG into optimization problems.

In conclusion, the proposed two-step EMG-and-optimization process has the advantages of being noninvasive, of requiring a single experimental session, and of using static optimization, thus making the proposed method easy to implement with acceptably low computational requirements. We think that EMG-andoptimization may offer a convenient way to estimate the muscle force for applications in various fields, including rehabilitation and sports biomechanics. In considering the observations by Erdemir et al. (2007), the generalization of the proposed method 
might be of particular interest in clinical biomechanics, e.g., to investigate neural control and to improve diagnosis or surgery planning.

\section{Conflict of interest}

We wish to confirm that there are no known conflicts of interest associated with this publication and there has been no significant financial support for this work that could have influenced its outcome.

\section{References}

Amarantini, D., Martin, L., 2004. A method to combine numerical optimization and EMG data for the estimation of joint moments under dynamic conditions. Journal of Biomechanics 37, 1393-1404.

Anderson, F.C., Pandy, M.G., 2001. Static and dynamic optimization solutions for gait are practically equivalent. Journal of Biomechanics 34, 153-161.

Billot, M., Simoneau, E., Van Hoecke, J., Martin, A., 2009. Coactivation at the ankle joint is not sufficient to estimate agonist and antagonist mechanical contribution. Muscle and Nerve, in press, doi:10.1002/mus.21530.

Buchanan, T.S., 1995. Evidence that maximum muscle stress is not a constant: differences in specific tension in elbow flexors and extensors. Medical Engineering and Physics 17, 529-536.

Buchanan, T.S., Lloyd, D.G., Manal, K., Besier, T.F., 2004. Neuromusculoskeletal modeling: estimation of muscle forces and joint moments and movements from measurements of neural command. Journal of Applied Biomechanics 20, 367-395.

Centomo, H., Amarantini, D., Martin, L., Prince, F., 2007. Muscle adaptation patterns of children with a trans-tibial amputation during walking. Clinical Biomechanics 22, 457-463.

Challis, J.H., 1997. Producing physiologically realistic individual muscle force estimations by imposing constraints when using optimization techniques. Medical Engineering and Physics 19, 253-261.

Chao, E.Y., Rim, K., 1973. Application of optimization principles in determining the applied moments in human leg joints during gait. Journal of Biomechanics 6 , 497-510.

Cholewicki, J., McGill, S.M., 1994. EMG assisted optimization: a hybrid approach for estimating muscle forces in an indeterminate biomechanical model. Journal of Biomechanics 27, 1287-1289.

Cholewicki, J., McGill, S.M., 1995. Comparison of muscle forces and joint load from an optimization and EMG assisted lumbar spine model: towards development of a hybrid approach. Journal of Biomechanics 28, 321-331.

Doorenbosch, C.A., Harlaar., J., 2003. A clinically applicable EMG-force model to quantify active stabilization of the knee after a lesion of the anterior cruciate ligament. Clinical Biomechanics 18, 142-149.

Dowling, J.J., 1997. The use of electromyography for the noninvasive prediction of muscle forces. Current issues. Sports Medicine 24, 82-96.

Erdemir, A., Mclean, S., Herzog, W., Van Den Bogert, A.J., 2007. Model-based estimation of muscle forces exerted during movements. Clinical Biomechanics 22, 131-154.
Isear Jr., J.A., Erickson, J.C., Worrell, T.W., 1997. EMG analysis of lower extremity muscle recruitment patterns during an unloaded squat. Medicine and Science in Sports and Exercise 29, 532-539.

Jinha, A., Ait-Haddou, R., Herzog, W., 2006. Predictions of co-contraction depend critically on degrees-of-freedom in the musculoskeletal model. Journal of Biomechanics 39, 1145-1152.

Lloyd, D.G., Besier, T.F., 2003. An EMG-driven musculoskeletal model to estimate muscle forces and knee joint moments in vivo. Journal of Biomechanics 36 765-776

Manal, K., Roberts, D.P., Buchanan, T.S., 2006. Optimal pennation angle of the primary ankle plantar and dorsiflexors: variations with sex, contraction intensity, and limb. Journal of Applied Biomechanics 22, 255-263.

Narici, M.V., Binzoni, T. Hiltbrand, E., Fasel, J., Terrier, F., Cerretelli, P. 1996. In vivo human gastrocnemius architecture with changing joint angle at rest and during graded isometric contraction. The Journal of Physiology 496 287-297.

Neptune, R.R., Wright, I.C., Van Den Bogert, A.J., 2000. A method for numerical simulation of single limb ground contact events: application to heel-toe running. Computer Methods in Biomechanics and Biomedical Engineering 3, $321-334$

Olney, S.J., Winter, D.A., 1985. Predictions of knee and ankle moments of force in walking from EMG and kinematic data. Journal of Biomechanics 18, 9-20.

Pincivero, D.M., Gandhi, V., Timmons, M.K., Coelho, A.J., 2006. Quadriceps femoris electromyogram during concentric, isometric and eccentric phases of fatiguing dynamic knee extensions. Journal of Biomechanics 39, 246-254.

Pincivero, D.M. Coelho, A.J. Campy, R.M. 2008. Contraction mode shift in quadriceps femoris muscle activation during dynamic knee extensor exercise with increasing loads. Journal of Biomechanics 41, 3127-3132.

Prilutsky, B.I., Gregor, R.J., 1997. Strategy of coordination of two- and one-joint leg muscles in controlling an external force. Motor Control 1, 91-115.

Raikova, R.T., Prilutsky, B.I., 2001. Sensitivity of predicted muscle forces to parameters of the optimization-based human leg model revealed by analytica and numerical analyses. Journal of Biomechanics 34, 1243-1255.

Rao, G., Amarantini, D., Berton, E., 2009. Influence of additional load on the moments of the agonist and antagonist muscle groups at the knee joint during closed chain exercise. Journal of Electromyography and Kinesiology 19, 459466.

Rasmussen, J., Damsgaard, M., Voigt, M., 2001. Muscle recruitment by the min/max criterion - a comparative numerical study. Journal of Biomechanics 34, 409415.

Seth, A., Pandy, M.G., 2007. A neuromusculoskeletal tracking method for estimating individual muscle forces in human movement. Journal of Biomechanics 40, 356-366.

Spagele, T., Kistner, A., Gollhofer, A., 1999. A multi-phase optimal contro technique for the simulation of a human vertical jump. Journal of Biomechanics 32, 87-91.

Toutoungi, D.E., Lu, T.W Leardini, A Catani, F, O'Connor, J., 2000. Cruciate ligament forces in the human knee during rehabilitation exercises. Clinical Biomechanics 15, 176-187.

Vigouroux, L., Quaine, F., Labarre-Vila, A., Amarantini, D., Moutet, F., 2007. Using EMG data to constrain optimization procedure improves finger tendon tension estimations during static fingertip force production. Journal of Biomechanics 40, 2846-2856.

Visser, J.J., Hoogkamer, J.E., Bobbert, M.F., Huijing, P.A., 1990. Length and moment arm of human leg muscles as a function of knee and hip-joint angles. European Journal of Applied Physiology and Occupational Physiology 61, 453-460. 